\title{
Glomerular Disease Education Experience across Nephrology Fellowship Programs: An International Survey
}

\author{
Harish Seethapathy ${ }^{a}$ Sayna Norouzi ${ }^{b}$ Kate J. Robson ${ }^{c, d}$ Lida Gharibvand \\ Ali Poyan Mehr ${ }^{f}$ \\ ${ }^{a}$ Division of Nephrology, Department of Medicine, Massachusetts General Hospital, Boston, MA, USA; ${ }^{b}$ Division \\ of Nephrology, Department of Medicine, Loma Linda University Medical Center, Loma Linda, CA, USA; 'Monash \\ University Department of Medicine, Centre for Inflammatory Diseases, Monash Medical Centre, Monash University, \\ Clayton, VIC, Australia; 'Department of Nephrology, Monash Health, Clayton, VIC, Australia; eLoma Linda University, \\ School of Allied Health Professions, Loma Linda, CA, USA; fDepartment of Nephrology, Kaiser Permanente San \\ Francisco Medical Center, San Francisco, CA, USA
}

\section{Keywords}

Glomerular disease - Glomerulonephritis · Education .

Nephropathology

\begin{abstract}
Introduction: Glomerulonephritis (GN) education is an important, albeit a challenging, component of nephrology fellowship training. We hypothesized that trainee experience varies widely across programs, leading to differences in selfreported competency levels in the diagnosis and management of glomerular diseases. Methods: The Glomerular Disease Study \& Trial Consortium (GlomCon) conducted an anonymous online survey to determine the educational experience of nephrology trainees. We used multiple-choice questions to obtain data about (a) curriculum-based education, (b) dedicated specialty clinic, and (c) exposure to pathology. We leveraged a visual analog scale of 1-100 (with a higher number indicating a higher comfort level) to assess self-reported levels of clinical competency. The survey was disseminated via email to the subscribing members of GlomCon and through Twitter. Results: In total, there were 109 respondents to our survey, and $56 \%$ were from training programs in the USA. Exposure to a specialized GN clinic was
\end{abstract}

karger@karger.com www.karger.com/gdz

Karger"
(C) 2021 The Author(s)

Published by S. Karger AG, Basel

This is an Open Access article licensed under the Creative Common Attribution-NonCommercial-4.0 International License (CC BY-NC) (http://www.karger.com/Services/OpenAccessLicense), applicable to the online version of the article only. Usage and distribution for commercial purposes requires written permission. reported by $45 \%$, while $77 \%$ reported the presence of an onsite nephropathologist at their training program. Self-reported competency scores were $59 \pm 25$ and $52 \pm 25$ for diagnosis and treatment of glomerular diseases, respectively. Days spent in a GN clinic per year, years of fellowship, and dedicated nephropathology didactics were associated with higher diagnosis and treatment competency scores. Conclusion: Trainees report a wide variation in glomerular disease education across fellowship programs. A lack of nephropathology exposure and a dedicated GN curriculum was associated with lower scores in self-reported clinical competency in caring for patients with glomerular disease.

(C) 2021 The Author(s).

Published by S. Karger AG, Basel

\section{Introduction}

Since the late 1950s, when "Metabolism" split into two distinct fields to establish today's Nephrology and Endocrinology, glomerular disease education has been an in-

Harish Seethapathy and Sayna Norouzi share equal contribution (cofirst authors). 
tegral part of the nephrology fellowship training in the USA. The study of glomerular diseases is mandated as a core component of the fellowship curriculum by the Accreditation Council for Graduate Medical Education and the American Society of Nephrology $[1,2]$. Nondiabetic glomerular diseases account for more than $25 \%$ of the cases of chronic kidney disease worldwide and are the third leading cause of end-stage kidney disease in the USA [3-5]. Nondiabetic glomerular diseases, as a cause of chronic kidney disease and end-stage kidney disease, are particularly more common in young adults, leading to significant morbidity and mortality over time [6]. The management of glomerular diseases is frequently a multidisciplinary team effort and involves a comprehensive understanding of disease pathogenesis, clinical presentation, nephropathology, and the use of immunosuppressive medications that carry considerable adverse effect risks. Therefore, it is vital that trainees entering independent practice are adequately trained in the management of patients with glomerular diseases.

Although some centers have specialized glomerulonephritis (GN) clinics, an onsite nephropathology department, and dedicated GN training programs that offer specialized glomerular disease education, many training programs lack the presence of an onsite nephropathologist and may not have sufficient patient volume to apportion time and faculty for the sole purpose of a GN clinic. In this study, we hypothesized that the exposure to glomerular disease education likely varies significantly across nephrology training programs. Through the Glomerular Disease Study \& Trial Consortium (GlomCon), we conducted an anonymous survey of nephrology fellows worldwide to assess the association between the selfreported competency of nephrology fellows in diagnosing and managing patients with glomerular diseases and the structure of their training program.

\section{Methods}

An online survey was distributed to subscribing members of GlomCon (3,521 email recipients) and also distributed via the social media platform, Twitter (6,647 followers for @GlomCon), in February 2020. The survey was jointly designed by the authors of this paper. In addition to collecting basic demographics, the survey questions were designed to determine the educational experience and self-reported competency across levels of nephrology trainees. Identifying features of respondents were not collected, and all responses were recorded anonymously. The survey targeted trainees of adult nephrology since the focus and training of glomerular diseases are vastly different between the curriculum of adult and pediatric fellowship programs. We invited fellows of all levels of training to participate, and the survey was not restricted by country or location of the participant. We used multiple-choice questions to obtain data about (a) curriculum-based education, (b) dedicated specialty clinic, and (c) exposure to pathology. Selfreported experiences were categorized by nephropathology exposure as follows: group A - no onsite nephropathologist(s) and insufficient exposure to renal pathology, group B - presence of onsite nephropathologist(s) but limited exposure and no formal didactics, group C - no onsite nephropathologist(s) but dedicated didactic sessions, and group D - presence of onsite nephropathologist(s) and dedicated didactic sessions.

We leveraged a Likert scale of 1-100 (with a higher number indicating a higher comfort level) to assess self-reported levels of clinical competency. Competency was categorized into low $(<50)$, medium (50-74), and high (75 and above) based on the scores. Frequency and percentages were used to describe categorical variables, and mean and standard deviation were used for continuous variables. A multivariable linear regression model was used to evaluate for predictors of high competency. All statistical analyses were performed using SAS 9.4 (SAS Institute Inc., Cary, NC, USA). All analyses were performed at an alpha level of 0.05 .

\section{Results}

In total, there were 109 respondents to our survey (Table 1). Respondents by the year of fellowship training were as follows: 27 (25\%) were first-year fellows (25\%), 36 (34\%) were in their second year while $24(22 \%)$ were in their third year $(22 \%)$, and $21(19 \%)$ were in their fourth year of training. Forty-one respondents (37\%) were identified as female, $64(59 \%)$ as male, and 4 (4\%) opted not to answer. Sixty-one respondents $(56 \%)$ were current nephrology fellows enrolled at fellowship programs in the USA, while 47 (44\%) were trainees enrolled at programs in other countries. Among other regions, Asia (42\%) had the most respondents. Within the USA, $36 \%$ of the responses came from trainees in the northeast, $33 \%$ from the south, $17 \%$ from the midwest, and 14\% from the west (Table 1).

\section{Glomerular Disease and Nephropathology Exposure at Training Programs}

Forty-nine (45\%) trainees reported the presence of a specialized glomerular disease clinic at their institution, with $51 \%(n=25)$ of those indicating the presence of a dedicated glomerular disease curriculum. Sixty (55\%) trainees reported no specialized GN clinic, while 32\% $(n=19)$ of those reported no specialty clinic or dedicated curriculum. Among those with exposure to a glomerular disease clinic $(n=46), 41 \%$ spent $\leq 10$ days per year while $59 \%$ spent $>10$ days per year in the GN clinic. The presence of an onsite nephropathologist was reported by $77 \%$ of the responders.
Glomerular Dis 2022;2:89-94 DOI: $10.1159 / 000521598$
Seethapathy/Norouzi/Robson/ Gharibvand/Mehr 
Table 1. Characteristics of survey respondents $(N=109)$

\begin{tabular}{ll}
\hline Characteristics & Frequency (\%) \\
\hline $\begin{array}{l}\text { Gender identity } \\
\text { Male }\end{array}$ & $64(59)$ \\
Female & $41(37)$ \\
Other/did not answer & $4(4)$ \\
Days spent in GN clinic & \\
$\quad \leq 10$ days/year & $82(75)$ \\
>10 days/year & $27(25)$ \\
Year of fellowship training & \\
1st year & \\
2nd year & $27(25)$ \\
3rd year & $36(33)$ \\
4th year & $24(22)$ \\
Training program exposure & $21(19)$ \\
Dedicated glomerular disease clinic & \\
Onsite nephropathologist & $49(46)$ \\
Location of fellowship programs & $82(77)$ \\
USA & \\
Northeast & $61(56)$ \\
South & $22(36)$ \\
Midwest & $20(33)$ \\
West and Pacific Islands & $11(17)$ \\
International & $7(14)$ \\
Nephropathology exposure* & $47(43)$ \\
Group A & \\
Group B & $11(10)$ \\
Group C & $14(13)$ \\
Group D & $16(15)$ \\
\hline & $68(62)$ \\
\hline
\end{tabular}

GN, glomerulonephritis. ${ }^{\S}$ Frequency does not add up to $100 \%$ due to missing data. *Training programs were defined for nephropathology exposure as follows: group A - no onsite nephropathologist(s) and insufficient exposure to renal pathology; group B - presence of onsite nephropathologist(s) but limited exposure and no formal didactics; group C - no onsite nephropathologist(s) but dedicated didactic sessions; group D presence of onsite nephropathologist(s) and dedicated didactic sessions.

\section{Self-Reported Competency Levels in Diagnosis and}

Management of Glomerular Diseases

In a visual analog scale (1-100), the mean and standard deviation of self-reported competency for GN diagnosis and treatment were $59 \pm 25$ and $52 \pm 25$, respectively.

\section{Predictors of Self-Reported Competency}

The number of days spent in a GN clinic per year was higher for trainees with higher self-competency for both diagnosis $(15 \%$ with $p<0.01)$ and treatment $(16 \%$ with $p<0.01)$ (Table 2). Trainees in group C $(23 \%, p<0.01)$ and group $\mathrm{D}(27 \%, p<0.01)$ programs reported higher diagnosis competency scores when compared to trainees in group A (no onsite nephropathologist(s) and insuffi- cient exposure to renal pathology), which had the lowest self-reported competency scores. Diagnosis competency scores were similar for trainees in group A and group B $(p=0.19)$ (Table 2$)$. Similarly, trainees in group C $(30 \%$, $p<0.01)$ and group D $(20 \%, p<0.01)$ programs reported higher competency scores for treatment when compared to trainees in group A. Diagnosis competency scores were similar for trainees in group A and group B $(p=0.15)$ (Table 2).

\section{Supplemental Education Avenues}

Among the 109 respondents, 75 (69\%) reported supplementing their glomerular disease knowledge using GlomCon resources, while 34 (31\%) did not use GlomCon or were not aware that the platform existed. Eight respondents $(7 \%)$ did not feel that they would benefit from enrolling in a virtual glomerular fellowship, 9 would consider based on time commitments or curriculum format, while $92(84 \%)$ would be interested in such a program. Among the 8 trainees who were not interested in an online program, all had exposure to a dedicated glomerular disease clinic or curriculum at their current training program. Fifty-five percent of trainees who had exposure to a dedicated glomerular disease clinic or curriculum felt that they would benefit from additional training in the form of an online fellowship.

\section{Discussion}

We designed a survey to determine the exposure and competency of trainees to glomerular diseases. Our results suggest several key areas that training programs may focus on to further enhance the clinical competency of their trainees. We found that trainees from programs equipped with a specialized glomerular disease clinic reported a higher level of competency for both diagnosis and treatment of glomerular diseases, while trainees that were not exposed to an onsite nephropathologist(s) and reported insufficient exposure to renal pathology had the lowest scores. In an era of increasing sub-specialization $[7,8]$, there has been an inception of advanced nephrology training programs across the USA. While kidney transplantation fellowship programs have existed for decades, recent years have seen an increasing interest in further sub-specialty training offered in various niches of nephrology such as onconephrology, home dialysis, interventional nephrology, and cardionephrology [9-11]. While there are only two glomerular disease fellowship programs in the USA, many programs have established 


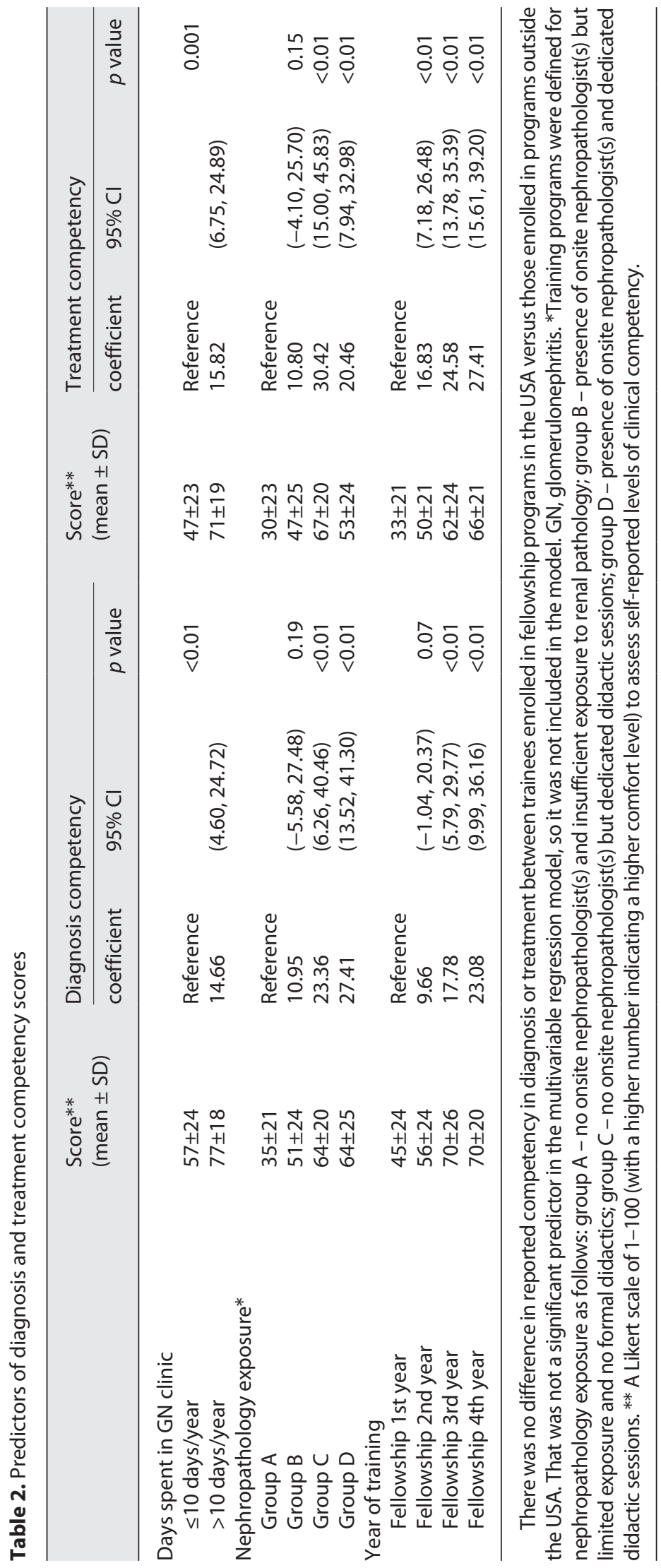

dedicated glomerular disease clinics, with $45 \%$ of respondents reporting the presence of such at their training programs. While there may be faculty who are interested in glomerular disease in many institutions and may dedicate more clinic time to managing such patients, the establishment of a dedicated glomerular disease clinic provides the trainee a more focused and comprehensive experience and also affords the opportunity to develop a dedicated curriculum. Programs with such dedicated clinics are also more likely to be attractive to future fellowship applicants.

In our study, there was also a linear relationship between trainee competency levels and number of days spent in a glomerular disease clinic per year. The vast majority of the nephrology fellowship curriculum focuses on inpatient management, and most fellows are assigned general nephrology continuity clinics during training. As shown in our study, fellows who spend more than 10 days a year in a glomerular disease clinic report higher levels of competency. Programs and fellows should differentiate and choose between creating "awareness" and developing “ competency." In many programs, fellows rotate through the glomerular disease clinic for a few days a year. While this may provide an idea of what managing such patients may entail, developing competency requires spending 6 months to a year following patients in a glomerular disease clinic. Developing skills in the nuances of glomerular disease management, including the use of immunosuppression, requires a more extensive education that may not be possible in a short duration. Our results draw parallels to a similar study of 76 trainees on self-reported home dialysis competency, where despite the majority of respondents (59\%) reporting exposure to a home hemodialysis clinic, $67 \%$ of respondents reported no or minimal preparedness for managing home hemodialysis upon graduation [12].

Interpretation and use of nephropathology is a major component of glomerular disease management. In our survey, the presence of an onsite nephropathologist or an offsite nephropathologist with dedicated didactic sessions was associated with higher levels of competency with diagnosis and treatment. A 2015 study by Mechery et al. [13] reported that trainees in programs with a nephropathology division were 3 times more likely to be satisfied with their nephropathology education. In the same study, only $61 \%$ of the respondents were satisfied with their nephropathology experience, and $75 \%$ of the respondents had only monthly didactic sessions. Interestingly, in our study, trainees reported more competency with the presence of dedicated didactic sessions even if 
the nephropathologist was offsite. These results, when combined with the results of our study, provide an important perspective on nephropathology exposure in ACGME nephrology training programs. The size of certain programs does not allow for dedicated onsite nephropathology presence, but these results indicate that structuring dedicated didactics irrespective of the location of the nephropathologist and hence total time spent learning nephropathology with an expert can provide a satisfying educational experience for trainees.

Our results differ somewhat from a previous study by Berns et al. [14]. In that survey of 133 recent fellowship graduates in which self-reported competency was recorded for all major components of fellowship training, $>80 \%$ reported being well-trained in glomerular diseases. However, around 53\% reported inadequate nephropathology exposure and did not feel competent in the interpretation of nephropathology. The reasons for the differences in results are twofold. One, our study focused exclusively on glomerular diseases, and hence respondents likely tended to determine their experience in a more precise manner. Two, our study included trainees at all levels of training, and early trainees are more likely to perceive lower levels of competency when compared to advanced trainees.

Our study has several strengths. This is the first and largest study to date that exclusively determines trainee experience with glomerular diseases. Our responses from across the world provide a global view of trainee experience, thereby increasing generalizability. The results of our study must be interpreted in the context of its limitations. Like all surveys, our study provides a subjective view and not an objective confirmation of competency. We included all levels of trainees, many of whom are still learning and are in the process of acquiring skills for independent practice, and hence there was likely to be a leftward shift in reported competency. Our intention was to avoid recall bias and contamination of post-fellowship practice experience influencing the view of training experience.

Our study has multiple limitations. Our survey was mainly distributed on social media and via emails. Due to the nature of dissemination, we were unable to determine the response rate. Due to the anonymous nature of our survey, we were not able to verify trainee credentials or ensure representative sample across all programs. Our respondents may disproportionately comprise trainees with a particular interest in glomerular disease, or represent those who may have a disproportionately lower exposure to GN education during training, or who may perceive their training as insufficient. Last, the respondents are enriched for those who may follow GlomCon for its educational content. This may affect the generalizability of the study. This selection bias is particularly important to consider due to the anonymous, international nature of the survey, which prevents correlating our self-assessment to objective evaluations such as the American Society of Nephrology in-training scores for glomerular diseases. Further, our survey did not consider additional, more granular details concerning the individual training environment, such as biopsy conferences or pathology electives. Another limitation was a low total number of responses (109) which may have affected our results. The voluntary nature of the survey likely affected the response rate, as in other educational surveys of this kind. Our survey did not explicitly define the terms used in the survey questions, and the results should be interpreted as a confluence of subjective experiences and not an objective evaluation. The utilization of a high-range Likert scale (0-100) may have affected the precision of the results. Considering the design of our survey, the Dunning-Kruger effect may have led some participants with low abilities but situated in advanced centers with specialized clinics and dedicated pathologists to overestimate their competency.

We believe that our study identifies deficits in glomerular disease education that need to be addressed. Our survey was distributed through social media and GlomCon, and many of our respondents (69\%) use GlomCon to increase their knowledge of glomerular diseases. This shows that trainees are actively seeking out novel educational tools to improve their understanding and exposure to glomerular disease. Structural challenges and patient pool may be barriers for many programs wanting to implement specialized clinics or programs. In an era where remote and online learning is becoming increasingly common, more steps need to be taken to increase access to educational resources in challenging areas of nephrology, such as glomerular diseases. Taking the results of this survey into consideration, GlomCon is creating an online fellowship curriculum that will supplement the knowledge of trainees and increase their confidence and comfort in managing patients with glomerular disease (see online suppl. material; for all online suppl. material, see www.karger.com/doi/10.1159/000521598).

\section{Statement of Ethics}

Ethical approval was not required for this study in accordance with local/national guidelines. Written informed consent from participants was not required in accordance with local/national guidelines. 


\section{Conflict of Interest Statement}

H.S. was supported by a fellowship grant from Relypsa Inc. (2019-2020). The authors have no other conflicts of interest to declare.

\section{Funding Sources}

There was no funding..

\section{Author Contributions}

Conceptualization: H.S., S.N., K.J.R., L.G., and A.P.M. Data analysis and interpretation: H.S., S.N., K.J.R., L.G., and A.P.M. Manuscript draft and re-writing: H.S., S.N., K.J.R., L.G., and A.P.M.

\section{Data Availability Statement}

Data used for analysis shall be made available to the journal upon formal request. All data analyzed during this study are included in this article. Further enquiries can be directed to the corresponding author.

\section{References}

1 Kumar R, Alpern R, Berl T, Blantz R, Chevalier R, Hostetter T, et al. Nephrology core curriculum. The American society of nephrology and the American society of nephrology training program directors committee. J Am Soc Nephrol. 1997;8:1018-27.

2 Accreditation Council for Graduate Medical Education. ACGME program requirements for graduate medical education in nephrology; 2020.

3 Floege J, Barbour SJ, Cattran DC, Hogan JJ, Nachman PH, Tang SCW, et al. Management and treatment of glomerular diseases (part 1): conclusions from a kidney disease: improving global outcomes (KDIGO) controversies conference. Kidney Int. 2019;95(2):268-80.

4 Rovin BH, Caster DJ, Cattran DC, et al. Management and treatment of glomerular diseases (part 2): conclusions from a Kidney disease: improving global outcomes (KDIGO) controversies conference. Kidney Int. 2019;95(2):281-95.
5 Kidney Disease Improving Global Outcomes (KDIGO). Controversies conference on glomerular diseases; 2017. p. 1-17.

6 United States Renal Data System. 2018 USRDS annual data report: Epidemiology of kidney disease in the United States. Bethesda: National Institutes of Health, National Institute of Diabetes and Digestive and Kidney Diseases; 2018.

7 Dalen JE, Ryan KJ, Alpert JS. Where have the generalists gone? They became specialists, then subspecialists. Am J Med. 2017;130(7): 766-8.

8 West CP, Dupras DM. General medicine versus subspecialty career plans among internal medicine residents. JAMA. 2012;308(21): 2241-7.

9 Munshi R, Winrow RM, Wu JS, Treit K, Bieber SD. Advanced dialysis fellowship. Hemodial Int. 2014;18(1):S52-4.
10 Rangaswami J, Mathew RO, McCullough PA. Resuscitation for the specialty of nephrology: is cardionephrology the answer? Kidney Int. 2018;93(1):25-6.

11 Rosenberg ME. Adult nephrology fellowship training in the United States: trends and issues. J Am Soc Nephrol. 2007;18(4):1027-33.

12 Gupta N, Taber-Hight EB, Miller BW. Perceptions of home dialysis training and experience among US nephrology fellows. Am J Kidney Dis. 2021 May;77(5):713-8.e1.

13 Mechery V, Hernandez T, Mathew AT, Wanchoo R, Seshan SV, Jhaveri KD, et al. Nephropathology education during nephrology fellowship training in the United States. Kidney Int Rep. 2018;3(2):236-41.

14 Berns JS. A survey-based evaluation of selfperceived competency after nephrology fellowship training. Clin J Am Soc Nephrol. 2010;5(3):490-6. 